\title{
Towards optimizing use of PLA2R antibody testing in membranous nephropathy
}

\author{
Jonathan J. Hogan ${ }^{1} \cdot$ Jarcy Zee ${ }^{2,3} \cdot$ Laurence H. Beck ${ }^{4}$
}

Published online: 27 January 2021

(c) Italian Society of Nephrology 2021

The ability to detect and measure autoantibodies to the target antigen M-type phospholipase A2 receptor (PLA2R) in patients with membranous nephropathy $(\mathrm{MN})$ has been revolutionary for understanding and diagnosing $\mathrm{MN}$, as well as monitoring humoral disease activity by following the trend of PLA2R-Ab titers. The definition of PLA2R-Ab seropositivity can vary depending on the specific assay used and its reference range. There are two FDA-approved commercial assays available for PLA2R-Ab: a cell-based indirect immunofluorescence test (IIFT) and an enzyme-linked immunosorbent assay (ELISA), both of which detect total $\mathrm{IgG}$ against PLA2R [1]. According to manufacturer instructions for the IIFT, a positive signal at 1:10 defines seropositivity. For the ELISA, a clear positive assay is represented by a titer $>20 \mathrm{RU} / \mathrm{mL}$, while a titer in the $14-20 \mathrm{RU} / \mathrm{mL}$ range is considered borderline, and $<14 \mathrm{RU} / \mathrm{mL}$ is considered negative. These thresholds were established based on the study by Dahnrich et al. that included 200 cases of primary MN, 230 other kidney diseases, 316 other autoimmune diseases, and 291 healthy individuals [1]. While many clinical laboratories and most publications in the field use these definitions for seropositivity, several studies have shown that using a much lower ELISA cutoff (2-3 RU/mL) increases sensitivity without sacrificing specificity, and therefore more accurately defines seropositivity [2-5].

Jonathan J. Hogan

jonathan.hogan2@pennmedicine.upenn.edu

1 Division of Nephrology, Perelman School of Medicine, Hospital of the University of Pennsylvania, 3400 Spruce Street, 1 Founders, Philadelphia, PA 19104, USA

2 Department of Biostatistics, Epidemiology, and Informatics, Perelman School of Medicine, University of Pennsylvania, Philadelphia, PA, USA

3 Children's Hospital of Philadelphia, Philadelphia, PA, USA

4 Division of Nephrology, Boston University School of Medicine and Boston Medical Center, Boston, MA, USA
In this issue of the Journal of Nephrology, Porcelli et al. re-assess the test characteristics of the commercial ELISA for PLA2R-Ab in a cohort of 495 consecutive Caucasian patients from several nephrology units in Italy [6]. All patients underwent kidney biopsy and provided baseline serum samples prior to initiating any immunosuppression. Idiopathic MN was diagnosed in 126 patients, while the remaining 369 had other disorders. Using the biopsy diagnosis of idiopathic MN as gold standard for "disease," the sensitivity, specificity, diagnostic efficiency, and positive and negative predictive values of the PLA2R-Ab ELISA were calculated and an optimal ELISA threshold for seropositivity was calculated as $2.7 \mathrm{RU} / \mathrm{mL}$. Based on this reference range, 105 of the $126 \mathrm{MN}$ cases were characterized as PLA2R-associated (mean titer of $82 \mathrm{RU} / \mathrm{mL}$, interquartile range 21-215 RU/mL). The authors then compared baseline characteristics of PLA2R-Ab positive vs. PLA2R-Ab negative cases and found that PLA2R positive patients were older (mean age 60 vs. 52, p=0.028) and had higher estimated glomerular filtration rate $\left(74 \mathrm{vs.} .40 \mathrm{~mL} / \mathrm{min} / 1.73 \mathrm{~m}^{2}\right.$, $\mathrm{p}=0.533$ ). Serum albumin was near normal in the seronegative cases (mean 3.95 vs. $2.2 \mathrm{~g} / \mathrm{dL}$ seropositive cases, $\mathrm{p}=0.004$ ) despite similar levels of baseline proteinuria. The authors conclude that the optimal cutoff to provide increased sensitivity is within the $2.0-2.7 \mathrm{RU} / \mathrm{mL}$ range, and the best clinical performance based on positive likelihood ratio and diagnostic odds ratio is at $14 \mathrm{RU} / \mathrm{mL}$.

This study and others that have suggested revising the reference range for seropositivity in PLA2R-MN have important clinical implications. Using a lower reference range would increase sensitivity for using PLA2R antibody as a diagnostic biomarker as has been suggested by others. A study by Bobart et al. found that PLA2R-Ab results of 2-20 $\mathrm{RU} / \mathrm{mL}$, in the additional presence of a positive IIFT, were highly sensitive and specific for the diagnosis of PLA2R$\mathrm{MN}$ in patients with eGFR $>60 \mathrm{~mL} / \mathrm{min} / 1.73 \mathrm{~m}^{2}[2,5]$. An important caveat from this study is that $42.5 \%$ of patients with PLA2R-Ab within the $2-20 \mathrm{RU} / \mathrm{mL}$ range but with a 
negative IIFT had a pathological lesion other than MN on biopsy. Future validation of studies such as this and the current study by Porcelli et al., in addition to more widespread availability of the tests, will determine if PLA2R-Ab might eventually replace kidney biopsy as the gold standard for diagnosing PLA2R-MN. For the moment, we highly recommend that PLA2R-Ab serologies, especially when antibody titers are low or undetectable, be accompanied by PLA2R antigen staining of the kidney biopsy.

A pertinent question is therefore the relevance of a lowtiter PLA2R-Ab in diagnosing MN. In nephrotic syndrome that has yet to be characterized by kidney biopsy, it would be important to confirm such a low-titer PLA2R-Ab ELISA result via another serological method such as IIFT before a presumptive diagnosis of MN could be made. Alternatively, a clinician could retest a patient several months later to determine if the PLA2R-Ab ELISA titer increases into the clearly positive range with ongoing immunological disease activity. There are occasional false positives by ELISA in which the IIFT can be helpful to exclude the actual presence of PLA2R-Ab. The IIFT is helpful in this setting because the assay includes a negative control (untransfected HEK293 cells) that will detect non-specific IgG binding and therefore yield an indeterminate assay. In the ELISA, there are no negative control wells for an individual patient sample, so any $\operatorname{IgG}$ that non-specifically (i.e. not necessarily binding to PLA2R) adheres to the ELISA plate could lead to an apparent positive test for PLA2R-Ab. It is not clear whether or not the chance of this low-level false positivity on ELISA increases when lower thresholds are used.

A perhaps more commonly-encountered clinical scenario involves the monitoring of patients with known PLA2R$\mathrm{Ab}$ seropositivity as they undergo immunologic disease remission, either spontaneously or following immunosuppressive treatment. In these cases, it is critically important to follow the PLA2R-Ab until it becomes completely negative, that is $<2 \mathrm{RU} / \mathrm{mL}$ and possibly in combination with a negative IIFT. Concluding that a patient has achieved immunological remission when the PLA2R-Ab titer is in the 2-14 RU/mL is risky, as such low but detectable titers may represent persistence of active disease that requires further treatment or monitoring.

There are several issues that deserve consideration when defining optimal PLA2R-Ab test characteristics. In particular, the context in which the sensitivity of PLA2R-Ab testing is being determined requires specification. Sensitivity is defined as the proportion of those with a true positive test amongst all those with the "disease" who undergo testing. In the case of MN, the denominator ("disease") would be all patients with the histopathologic lesion of MN on biopsy. This would include PLA2R-positive MN (characterized by positive PLA2R-staining on kidney biopsy), secondary $\mathrm{MN}$, and cases of PLA2R-negative MN by biopsy staining for which secondary causes have been excluded. This latter group would also include patients with thrombospondin type-1 domain-containing 7A (THSD7A)-, NELL1- and semaphorin 3B-associated MN.

As PLA2R-associated MN represents approximately $80 \%$ of primary $\mathrm{MN}$, and primary $\mathrm{MN}$ represents $70-75 \%$ of all those found to have the pathologic lesion of MN on biopsy (the remainder reflecting secondary causes), PLA2R-associated disease should only represent 55-60\% of all MN (Fig. 1). Additionally, the percentage of MN patients with positive PLA2R-Ab would be expected to be lower than $55-60 \%$, since a proportion of such cases will be seronegative at the time of biopsy by virtue of having already entered immunologic
Fig. 1 Expected proportions of biopsy-proven $\mathrm{MN}$ by primary vs. secondary MN, PLA2Rassociated $\mathrm{MN}$, and seropositive or seronegative PLA2R-associated MN. (Asterisk) Although most seropositive PLA2R-associated $\mathrm{MN}$ cases are presumed to be primary $\mathrm{MN}$, there have been reports that entities that would typically be considered secondary MN (e.g., NSAIDassociated, sarcoidosis-associated, or $\mathrm{HCV}$-associated $\mathrm{MN}$ ) can be positive for PLA2R-Ab. These cases are represented by the striped region. PLA2R, phospholipase $\mathrm{A} 2$ receptor; $\mathrm{MN}$, membranous nephropathy; NSAID, non-steroidal anti-inflammatory drug; $\mathrm{HCV}$, hepatitis $\mathrm{C}$ virus, $\mathrm{Ab}$, antibody
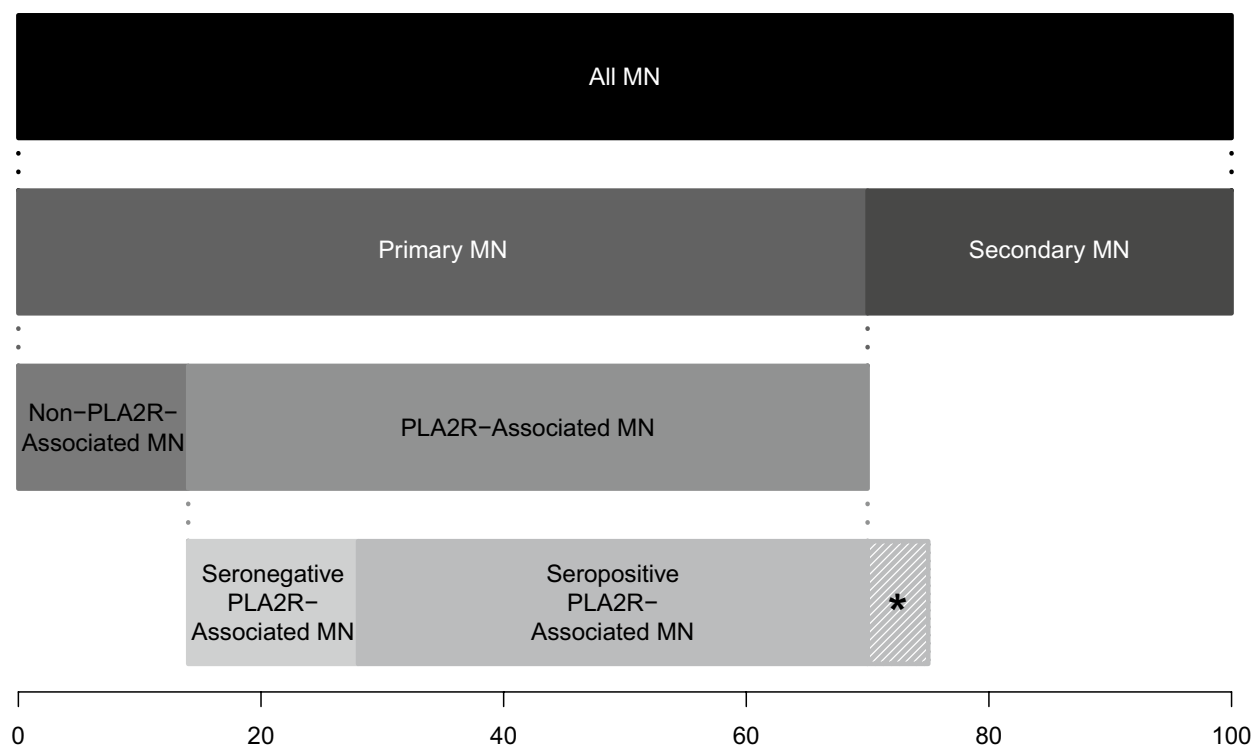

Percent 
remission. As examples, Qin et al. found a sensitivity of 75\% for PLA2R-Ab seropositivity in 572 Chinese MN cases, all of which were confirmed to have PLA2R positivity within immune deposits on kidney biopsy [7]. The seronegative patients in this cohort likely represent those who had already entered immunologic remission but still had proteinuria. Akiyama et al. found that only 53\% of the 100 idiopathic MN cases in their series had evidence of PLA2R-Ab [8]. It is likely that a proportion of this cohort had other types of MN (such as THSD7A-associated MN) and would never have possessed PLA2R-Ab. Thus, the sensitivity of the PLA2R-Ab ELISA depends not only on the threshold for positivity, but the context in which the test is being administered.

The study by Porcelli et al. has some important limitations that deserve mention when considering its impact on clinical application. First, the characterization of $\mathrm{MN}$ in the study did not include PLA2R antigen testing on kidney biopsy, which is currently the gold standard for making the diagnosis of PLA2R-associated MN. The lack of PLA2R antigen testing on biopsy confounds the interpretation of the PLA2R-Ab test characteristics in the study, the PLA2R-negative cohort might include PLA2R-associated MN cases who had already experienced spontaneous immunologic remission (suggested by the normalization of serum albumin) or those with other forms of MN. Second, there was no testing on kidney biopsy or serum for the presence for other autoantibody-antigen systems such as THSD7A or NELL1. Not characterizing the immunologic status of these cases in the context of biopsy staining also impacts the comparison of baseline clinical characteristics of PLA2R positive and negative cases in the study. Third, it is surprising that no cases of secondary MN were diagnosed in the study by Porcelli et al., as typically $25-30 \%$ of all MN represents secondary causes. Fourth, the optimal threshold of $2.7 \mathrm{RU} / \mathrm{mL}$ is based on the assumption that sensitivity and specificity are equally important metrics. As the authors mention, the clinical objective of the assay may imply a preference for other cutoffs depending on the costs of false negatives and false positive in an individual patient. In the study by Porcelli et al., using the lower cutoff of $2.0 \mathrm{RU} / \mathrm{mL}$ rather than $2.7 \mathrm{RU} / \mathrm{mL}$ as highly suggestive for MN would include more patients for screening, i.e., $16.7 \%$ of the current study sample; with low cost of additional screening, the benefits of correctly identifying more MN patients by serology alone would be relatively high.

Notwithstanding these limitations, the study by Porcelli et al. adds to a growing body of evidence advocating for the revision of reference ranges in PLA2R-Ab ELISA testing in MN. It is clear that using a lower cutoff for ELISA detection significantly decreases false negative tests and improves recognition of immunologically active disease. Until these reference ranges are changed to reflect these studies, it is important for clinicians to recognize these nuances to optimize the application of PLA2R-Ab testing in patient care.

\section{Compliance with ethical standards}

Conflict of interest JJH reports having received consulting fees from GSK, Alexion, Travere, Zyversa, Dimerix, Aurinia and Goldfinch Bio. $\mathrm{He}$ also reports salary support through grant funding as site principle investigator for studies from the National Institutes of Health, Gilead, GSK, Regeneron, Boehringer Ingelheim, Travere, Achillion, Calliditas, Omeros, and BMS. He receives royalties from UpToDate.com for topics written on calcium-phosphorous balance and monoclonal gammopathies of renal significance. He also is a co-inventor and has received royalties related to a pending patent involving the use of synthetic immunoreceptor engineered $\mathrm{T}$ cells for the treatment of membranous nephropathy. LHB has received research support from Sanofi Genzyme, Boston University Medical Center, is co-inventor US patent "Diagnostics for Membranous Nephropathy" with royalty income through Boston University, receives royalties from UpToDate.com for topic cards related to membranous nephropathy, has received served on advisory boards and received honoraria from Genentech, Visterra, Ionis, Alexion, and is a site PI on a clinical trial sponsored by Pfizer for novel therapeutics in FSGS. JZ has no conflicts of interests to declare.

Ethical approval This article does not contain any studies with human or animal subjects performed by any of the authors.

Informed consent For this type of study informed consent is not required.

\section{References}

1. Dahnrich C, Komorowski L, Probst C et al (2013) Development of a standardized ELISA for the determination of autoantibodies against human M-type phospholipase A2 receptor in primary membranous nephropathy. Clin Chim Acta 421:213-218

2. Timmermans SA, Damoiseaux JG, Heerings-Rewinkel PT et al (2014) Evaluation of anti-PLA2R1 as measured by a novel ELISA in patients with idiopathic membranous nephropathy: a cohort study. Am J Clin Pathol 142:29-34

3. Liu Y, Li X, Ma C et al (2018) Serum anti-PLA2R antibody as a diagnostic biomarker of idiopathic membranous nephropathy: the optimal cut-off value for Chinese patients. Clin Chim Acta 476:9-14

4. Tampoia M, Migliucci F, Villani C et al (2018) Definition of a new cut-off for the anti-phospholipase A2 receptor (PLA2R) autoantibody immunoassay in patients affected by idiopathic membranous nephropathy. J Nephrol 31:899-905

5. Bobart SA, De Vriese AS, Pawar AS et al (2019) Noninvasive diagnosis of primary membranous nephropathy using phospholipase A2 receptor antibodies. Kidney Int 95:429-438

6. Porcelli B, Guarnieri A, Ferretti F, et al. Diagnostic accuracy of anti-phospholipase A2 receptor (PLA2R) antibodies in idiopathic membranous nephropathy: an Italian experience. J Nephrol. 2020. https://doi.org/10.1007/s40620-020-00888-w.

7. Qin HZ, Zhang MC, Le WB et al (2016) Combined assessment of phospholipase A2 receptor autoantibodies and glomerular deposits in membranous nephropathy. J Am Soc Nephrol 27:3195-3203

8. Akiyama S, Akiyama M, Imai E, Ozaki T, Matsuo S, Maruyama S (2015) Prevalence of anti-phospholipase A2 receptor antibodies in Japanese patients with membranous nephropathy. Clin Exp Nephrol 19:653-660

Publisher's Note Springer Nature remains neutral with regard to jurisdictional claims in published maps and institutional affiliations. 